\title{
Classifications in Brief: Walch Classification of Primary Glenohumeral Osteoarthritis
}

\author{
Kiet V. Vo BA, BS, Daniel J. Hackett MD, Albert O. Gee MD, \\ Jason E. Hsu MD
}

Received: 20 November 2016/Accepted: 9 March 2017/Published online: 17 March 2017

(C) The Association of Bone and Joint Surgeons (B) 2017

\section{History}

Morphologic features in primary glenohumeral osteoarthritis do not appear in the same ways in all patients. Neer et al. [21] noted that posterior glenoid wear and posterior humeral head subluxation commonly occur in primary glenohumeral arthritis. Using linear measurements with CT, Friedman et al. [7] reported their observations of excessive glenoid retroversion. Despite acknowledgment of the various glenoid morphologic features and glenohumeral relationships that are associated with glenohumeral arthritis [7,21], it was only in 1999 that Walch et al. [29] classified these commonly observed features. In an effort to clarify etiology, progression, and treatment, Denard and Walch [4] and Walch et al. [29] used CT to classify morphologic features of the glenoid in primary glenohumeral osteoarthritis based on the concepts of glenoid version and the glenohumeral subluxation index.

\footnotetext{
Each author certifies that neither he, nor any member of his immediate family, have funding or commercial associations (consultancies, stock ownership, equity interest, patent/licensing arrangements, etc) that might pose a conflict of interest in connection with the submitted article.

All ICMJE Conflict of Interest Forms for authors and Clinical Orthopaedics and Related Research ${ }^{\circledR}$ editors and board members are on file with the publication and can be viewed on request.
}

K. V. Vo

University of Washington School of Medicine, Seattle, WA, USA

D. J. Hackett, A. O. Gee, J. E. Hsu ( $\square)$

Shoulder and Elbow Surgery, Department of Orthopaedics and Sports Medicine, University of Washington Medical Center, 1959 NE Pacific Street, Box 356500, Seattle, WA 98195-6500, USA

e-mail: jehsu@uw.edu

\section{Purpose}

Despite the relative success of shoulder arthroplasty in patients with primary glenohumeral arthritis, morphologic features of the glenoid have been considered a potential factor that may affect glenoid implant survival $[5,10]$ and patientreported outcomes [17]. Static posterior humeral head subluxation associated with preferential posterior wear of the glenoid has been reported to lead to decreased ROM, persistent pain, and decreased glenoid component survivorship after shoulder arthroplasty [8, 13]. Others have noted that implantation of a glenoid component in retroversion can result in increased risk of radiographic loosening [10].

Similar to other classification systems, the Walch classification may help guide appropriate surgical management, can facilitate communication among surgeons, and may provide prognostic information. The classification provides an anatomic descriptive characterization of primary glenohumeral osteoarthritis. However, the primary purpose of the Walch classification was to recognize that glenoid version and the relationship of the humeral head to the glenoid need to be considered during preoperative planning and surgery. With greater glenoid retroversion and posterior humeral head subluxation observed with posterior wear, Walch et al. suggested further attention should be given toward soft tissue balancing to restore the normal, anatomic relationship of the glenohumeral joint in the transverse plane [29].

\section{Description of the Walch Classification System}

Classification of morphologic features of the glenoid in the original Walch description is based on axial cuts of two- 
dimensional CT scans. Morphologic features of the glenoid are divided into three main groups with subtypes (Fig. 1) [29]. Type A is centered or symmetric arthritis without posterior subluxation of the humeral head. Type A1 has minor central wear or erosion, whereas Type A2 has severe or major central wear or erosion. Type B is characterized by asymmetric arthritis with posterior subluxation of the humeral head. Type B1 has no obvious glenoid erosion with posterior joint space narrowing, subchondral sclerosis, and osteophytes. Type B2 has apparent or obvious erosion of the posterior glenoid forming a biconcave appearance of the glenoid. Type $\mathrm{C}$ shows glenoid retroversion greater than $25^{\circ}$ (dysplastic in origin) regardless of glenoid erosion or the location of the humeral head with regard to the glenoid [15, 29].

This classification has implications for prosthetic replacement and may alter the surgeon's approach to glenoid reaming and selection of glenoid implant. In their study of 113 shoulders with primary glenohumeral arthritis, Walch et al. [29] found that patients with Type A arthritis had adequate stability for the prosthesis because of symmetric load distribution on the glenoid and absence of subluxation. Earlier reconstruction in symptomatic Type B glenohumeral arthritis was advised to restore normal anatomy and accurate placement of the prosthesis. Implant selection may be affected by preoperative characterization of morphologic features of the glenoid. As opposed to standard anatomic glenoid components for Type A glenoids, some surgeons have recommended augmented glenoid components and even reverse total shoulder arthroplasty for shoulders with substantial posterior wear and retroversion [4, 6, 26].

Walch et al. [29] suggested that the natural history of the Type B glenoid includes progressive posterior humeral head subluxation and glenoid retroversion as posterior wear increases. In severe cases of Type B morphologic features, fixed posterior subluxation necessitates more-complex soft tissue balancing or bony reconstruction to ensure concentricity of the humeral head component in the glenoid component during shoulder motion. Matsen et al. [19] and Walch et al. [30] cautioned that asymmetric load distribution on the posterior aspect of the glenoid component may predispose the patient to glenoid loosening through the rocking-horse effect.

\section{Validation}

Kidder et al. [15] performed a randomized, blinded study in which they examined intra- and interobserver reliability of the original Walch classification and a "regroup Walch classification" in which the original was simplified into three main groups of $\mathrm{A}, \mathrm{B}$, and $\mathrm{C}$ without subtypes (Table 1). The study showed excellent intraobserver reproducibility $(\kappa=0.90,0.93$, and 0.77 ) among the three observers. Despite excellent intraobserver reproducibility, interobserver reliability was only moderate $(\kappa=0.6)$.

Nowak et al. [23] also reported poor results regarding interobserver reliability. In their study, three attending shoulder surgeons and five shoulder and sports medicinetrained fellows independently and blindly evaluated CT scans of 26 consecutive patients with primary glenohumeral osteoarthritis. The overall interobserver agreement for all eight observers was moderate $(\kappa=0.508$; range, $0.181-0.714)$. The greatest interobserver agreement was found for Type B2 ( $\kappa=0.714$; substantial). They found moderate agreement for Types A2 $(\kappa=0.593)$ and $\mathrm{C}$ $(\kappa=0.575)$, fair agreement for Type A1 $(\kappa=0.273)$, and

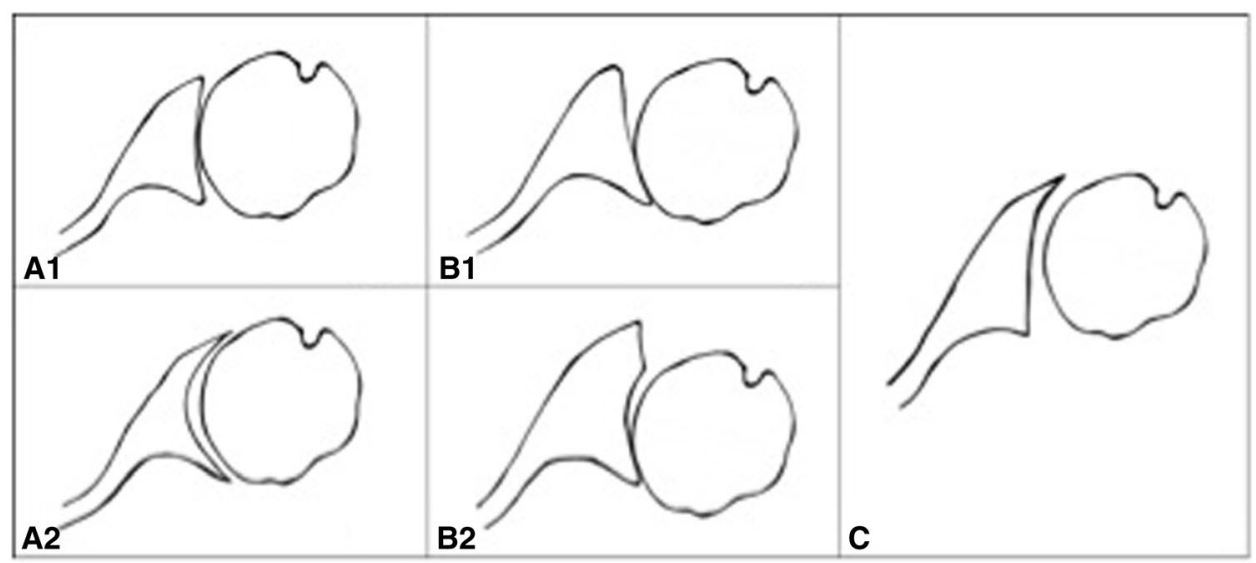

Fig. 1 The Walch classification [30] shows the morphologic features of the glenoid in primary glenohumeral arthritis. Reprinted from Karelse A, Leuridan S, Van Tongel A, Debeer P, Van Der Sloten J, Denis K, De Wilde LF. Consequences of reaming with flat and convex reamers for bone volume and surface area of the glenoid; a basic science study. J Orthop Surg Res. 2015;10:181. (http:// creativecommons.org/licenses/by/4.0/). 


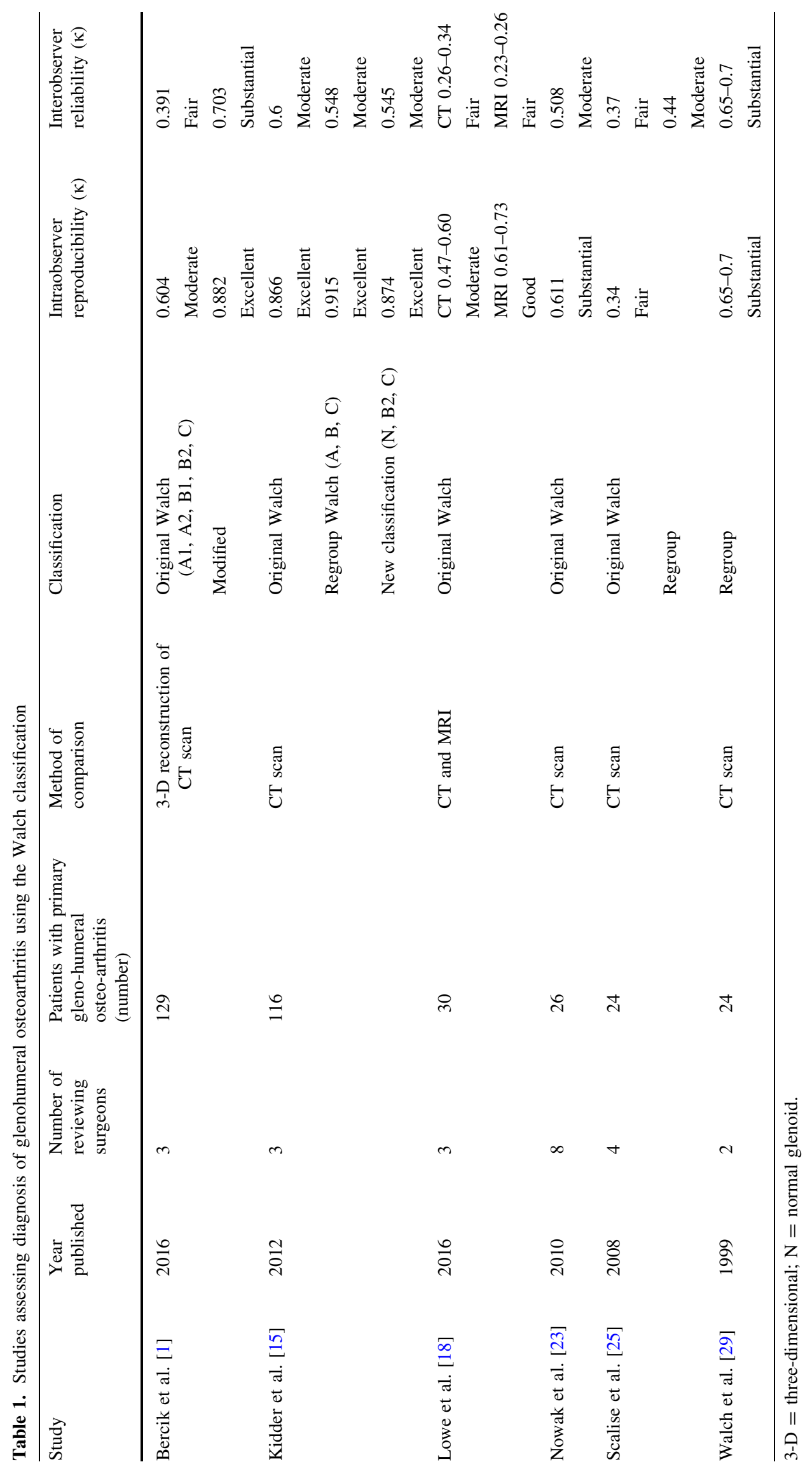


slight agreement for Type B1 $(\kappa=0.181)$. For intraobserver agreement, the overall $\kappa$ was 0.611 with a $\kappa$ range of 0.456-0.776, suggesting substantial reliability. No difference was found when comparing intraobserver results of fellows with those of attendings.

Scalise et al. [24] found only fair agreement among experienced shoulder surgeons using the Walch classification. In their study of 23 nonconsecutive patients with glenohumeral arthritis, four surgeons evaluated 24 shoulder CT scans in a randomized and blinded manner. Intra- and interobserver agreement were fair with $\kappa$ values of 0.37 and 0.34 , respectively. They found moderate $(\kappa=0.44)$ interobserver agreement when the classification system was simplified to three major categories without subtypes. Agreement for each major type was moderate for Types A $(\kappa=0.59)$ and $\mathrm{B}(\kappa=0.59)$ and excellent for Type $\mathrm{C}$ $(\kappa=0.89)$.

Lowe et al. [18] evaluated CT scans and MR images in 30 patients with primary glenohumeral osteoarthritis. Three fellowship-trained shoulder surgeons assessed glenoid version and the Walch classification. The interobserver reliability for CT $(\kappa=0.26-0.34)$ and MRI $(\kappa=0.23-$ $0.26)$ was only fair. Intraobserver reliability was moderate for CT $(\kappa=0.47-0.60)$ and good for MRI $(\kappa=0.61-$ 0.73). However, MRI overidentified Type $C$ glenoids and underidentified Type B2 glenoids, when compared with CT scans. Lowe et al. [18] suggested that MRI may lack adequate sensitivity to show a glenoid biconcavity.

Bercik et al. [1] examined intraobserver reproducibility and interobserver reliability using the original Walch classification and a modified Walch classification. In their study, three evaluators used the original Walch classification and modified classification to classify threedimensional (3-D) CT glenoid reconstructions of 129 nonconsecutive glenoids in a randomized and blinded manner. Their modified classification system included an addition of Type "B3" and "D" glenoids and a more precise definition of Type A2. Type B3 was defined as monoconcave with posterior wear and at least $15^{\circ}$ retroversion or at least $70 \%$ posterior subluxation of the humeral head, or both. Multiple biomechanical studies have shown that implantation of a glenoid component in greater than $15^{\circ}$ retroversion leads to peg perforation of the glenoid wall $[3,9,12,22]$. Type D glenoids were defined by Bercik et al. [1] as a glenoid with any level of anteversion or anterior subluxation of the humeral head. The Type A2 definition was updated to include glenoids in which a line drawn from anterior to posterior rims transects the humeral head, contrasting with Type A1, in which a line drawn from the anterior to posterior rim does not transect the humeral head [1].

For the original Walch classification, Bercik et al. [1] reported moderate intraobserver agreement $(\kappa=0.604)$ with $\kappa$ values of $0.624,0.629$, and 0.558 for Observers 1,2 , and 3 , respectively. In contrast to the original, there was excellent intraobserver agreement $(\kappa=0.882)$ with their modified Walch classification, with $\kappa$ values of 0.884 , 0.861 , and 0.9 for Observers 1, 2, and 3, respectively. They reported similar differences in interobserver agreement between the original and modified Walch classification. There was fair $(\kappa=0.391)$ and substantial $(\kappa=0.671)$ interobserver agreement for the original and modified Walch classifications, respectively.

\section{Limitations}

Although widely used since its introduction in 1999, the Walch classification has several limitations. Perhaps the most widely articulated criticism of the Walch classification is its inconsistent reproducibility among orthopaedic surgeons. Reported overall intraobserver agreement using the original Walch classification ranged from to fair $(\kappa=0.34)$ by Scalise et al. [24] to excellent $(\kappa=0.866)$ by Kidder et al. [15]. Interobserver agreement was even less reliable with a reported range from fair $(\kappa=0.37)$ by Scalise et al. [24] to moderate $(\kappa=0.600)$ by Kidder et al. [15]. This is in contrast to the substantial intra- and interobserver agreements (range, $\kappa=0.65-0.70$ ) reported by Walch et al. [29] in the original description of the classification system. Part of this discrepancy could be because in the original study, only the major categories of morphologic features of the glenoid (A, B, and C) were graded.

The potential overlap between Types $\mathrm{B}$ and $\mathrm{C}$ morphologic features of the glenoid is of particular controversy. While the original intent of the Walch classification may have been to characterize the morphologic features of Type B as that of progressive posterior glenoid wear and humeral head subluxation and Type $\mathrm{C}$ as that of dysplasia, the original description states that any glenoid with retroversion greater than $25^{\circ}$ should be considered Type C, "regardless of erosion." As a result, the Type C classification does not differentiate between a glenoid that acquired more than $25^{\circ}$ retroversion owing to progress of posterior wear versus a glenoid that had retroversion develop in a dysplastic fashion. The dysplastic glenoid often has distinct radiographic characteristics such as a hypoplastic scapular neck, rounding of the posteroinferior glenoid, and sometimes widened glenohumeral joint space owing to a hypertrophied posterior labrum and glenoid cartilage. The inability of the original Walch classification to distinguish between these distinct pathologic processes is of concern given that the prognosis and treatment options may be different.

As a result of this dilemma, expanded subcategories were introduced by Bercik et al. [1] and Nowak et al. [23]. 
This includes the B3 glenoid, which is described as a monoconcave glenoid with posterior bony wear and severe pathologic retroversion of at least $15^{\circ}$ or at least $70 \%$ posterior humeral head subluxation, or both [1]. This new description of morphologic features may have been inappropriately classified by Kidder et al. [15], Nowak et al. [23], and Scalise et al. [24], as Type C owing to severe retroversion or as Type A2 owing to the lack of biconcavity. The modified Walch classification can help prevent these potential areas of disagreement.

Additionally, the original description of the Walch classification does not address anterior or superior wear, rather focusing mainly on posterior wear. Bercik et al. [1] introduced a Type D glenoid as one with humeral head subluxation less than $40 \%$ or with any level of glenoid anteversion, but how common this variant is remains to be elucidated. These new categories may have implications on preoperative planning and treatment. For example, Bercik et al. [1] suggested that Type D glenoids should not be treated with standard shoulder arthroplasty owing to their increased risk of anterior instability.

Other criticisms of the Walch classification are its use of two-dimensional (2-D) CT scans to characterize a 3-D problem. With the increasing use and availability of 3-D reconstructions, it has been determined that they are more reliable for measurements and characterizing wear patterns than 2-D CT scans [2, 14, 16, 25]. Bercik et al. [1] found improved interobserver and intraobserver reliabilities to classify glenohumeral osteoarthritis when using 3-D glenoid reconstructions and the modified Walch classification. Additionally, Terrier et al. [27] reported that 3-D reconstructions may be particularly important for characterizing shoulders that have subluxation out of the plane of standard 2-D CT images (for example, those with posterosuperior subluxation) and that glenoid version is not highly correlated with glenohumeral subluxation.

Furthermore, studies have suggested that "functional malcentering" may occur only with the arm elevated $[11,28]$ such as seen on axillary radiographs [20] but not necessarily on CT scans with the arm at the side. Therefore, shoulders classified as Walch Type A based on a CT scan may posteriorly subluxate with arm elevation but will not be classified as a Walch Type B until glenoid wear creates static posterior subluxation with the arm at the side.

\section{Conclusions}

Classification of morphologic features of the glenoid in terms of posterior erosion and retroversion can have important clinical implications in the treatment of primary glenohumeral arthritis. Although the Walch classification is not as reliable as suggested in the original description, improvements to the classification might improve its reliability among observers, and it continues to be the most commonly used description of morphologic features of the glenoid. As our understanding of these morphologic features continues to evolve, additional investigation is needed to determine if further subtyping of the original classification improves its reliability and if 3-D reconstructions are required to accurately and reliably classify morphologic features.

\section{References}

1. Bercik MJ, Kruse K 2nd, Yalizis M, Gauci MO, Chaoui J, Walch G. A modification to the Walch classification of the glenoid in primary glenohumeral osteoarthritis using three-dimensional imaging. J Shoulder Elbow Surg. 2016;25:1601-1606.

2. Budge MD, Lewis GS, Schaefer E, Coquia S, Flemming DJ, Armstrong AD. Comparison of standard two-dimensional and three-dimensional corrected glenoid version measurements. $J$ Shoulder Elbow Surg. 2011;20:577-583.

3. Clavert P, Millett PJ, Warner JJ. Glenoid resurfacing: what are the limits to asymmetric reaming for posterior erosion? $J$ Shoulder Elbow Surg. 2007;16:843-848.

4. Denard PJ, Walch G. Current concepts in the surgical management of primary glenohumeral arthritis with a biconcave glenoid. J Shoulder Elbow Surg. 2013;22:1589-1598.

5. Farron A, Terrier A, Buchler P. Risks of loosening of a prosthetic glenoid implanted in retroversion. J Shoulder Elbow Surg. 2006;15:521-526.

6. Favorito PJ, Freed RJ, Passanise AM, Brown MJ. Total shoulder arthroplasty for glenohumeral arthritis associated with posterior glenoid bone loss: results of an all-polyethylene, posteriorly augmented glenoid component. J Shoulder Elbow Surg. 2016; 25:1681-1689.

7. Friedman RJ, Hawthorne KB, Genez BM. The use of computerized tomography in the measurement of glenoid version. $J$ Bone Joint Surg Am. 1992;74:1032-1037.

8. Gerber C, Costouros JG, Sukthankar A, Fucentese SF. Static posterior humeral head subluxation and total shoulder arthroplasty. J Shoulder Elbow Surg. 2009;18:505-510.

9. Gillespie R, Lyons R, Lazarus M. Eccentric reaming in total shoulder arthroplasty: a cadaveric study. Orthopedics. 2009;31:21.

10. Ho JC, Sabesan VJ, Iannotti JP. Glenoid component retroversion is associated with osteolysis. J Bone Joint Surg Am. 2013;95:e82.

11. Hsu JE, Gee AO, Lucas RM, Somerson JS, Warme WJ, Matsen FA 3rd. Management of intraoperative posterior decentering in shoulder arthroplasty using anteriorly eccentric humeral head components. J Shoulder Elbow Surg. 2016;25:1980-1988.

12. Iannotti JP, Greeson C, Downing D, Sabesan V, Bryan JA. Effect of glenoid deformity on glenoid component placement in primary shoulder arthroplasty. J Shoulder Elbow Surg. 2012;21:48-55.

13. Iannotti JP, Norris TR. Influence of preoperative factors on outcome of shoulder arthroplasty for glenohumeral osteoarthritis. $J$ Bone Joint Surg Am. 2003;85:251-258.

14. Jacxsens M, Van Tongel A, Henninger HB, De Coninck B, Mueller AM, De Wilde L. A three-dimensional comparative study on the scapulohumeral relationship in normal and osteoarthritic shoulders. J Shoulder Elbow Surg. 2016;25:16071615.

15. Kidder JF, Rouleau DM, DeFranco MJ, Pons-Villanueva J, Dynamidis S. Revisited: Walch classification of the glenoid in glenohumeral osteoarthritis. Shoulder Elbow. 2012;4:11-15. 
16. Kwon YW, Powell KA, Yum JK, Brems JJ, Iannotti JP. Use of three-dimensional computed tomography for the analysis of the glenoid anatomy. J Shoulder Elbow Surg. 2005;14:85-90.

17. Leschinger T, Raiss P, Loew M, Zeifang F. Predictors of medium-term clinical outcomes after total shoulder arthroplasty. Arch Orthop Trauma Surg. 2017; 137:187-193.

18. Lowe JT, Testa EJ, Li X, Miller S, DeAngelis JP, Jawa A. Magnetic resonance imaging is comparable to computed tomography for determination of glenoid version but does not accurately distinguish between Walch B2 and C classifications. $J$ Shoulder Elbow Surg. 2016 Oct 17. [Epub ahead of print] doi: 10. 1016/j.jse.2016.09.024.

19. Matsen FA 3rd, Clinton J, Lynch J, Bertelsen A, Richardson ML. Glenoid component failure in total shoulder arthroplasty. J Bone Joint Surg Am. 2008;90:885-896.

20. Matsen FA 3rd, Gupta A. Axillary view: arthritic glenohumeral anatomy and changes after ream and run. Clin Orthop Relat Res. 2014;472:894-902.

21. Neer CS 2nd, Watson KC, Stanton FJ. Recent experience in total shoulder replacement. J Bone Joint Surg Am. 1982;64:319-337.

22. Nowak DD, Bahu MJ, Gardner TR, Dyrszka MD, Levine WN, Bigliani LU, Ahmad CS. Simulation of surgical glenoid resurfacing using three- dimensional computed tomography of the arthritic glenohumeral joint: the amount of glenoid retroversion that can be corrected. $J$ Shoulder Elbow Surg. 2009;18:680-688.

23. Nowak DD, Gardner TR, Bigliani LU, Levine WN, Ahmad CS. Interobserver and intraobserver reliability of the Walch classification in primary glenohumeral arthritis. J Shoulder Elbow Surg. 2010;19:180-183.

24. Scalise JJ, Codsi MJ, Brems JJ, Iannotti JP. Inter-rater reliability of an arthritic glenoid morphology classification system. $J$ Shoulder Elbow Surg. 2008;17:575-577.

25. Scalise JJ, Codsi MJ, Bryan J, Brems JJ, Iannotti JP. The influence of three-dimensional computed tomography images of the shoulder in preoperative planning for total shoulder arthroplasty. J Bone Joint Surg Am. 2008;90:2438-2445.

26. Stephens SP, Spencer EE, Wirth MA. Radiographic results of augmented all-polyethylene glenoids in the presence of posterior glenoid bone loss during total shoulder arthroplasty. J Shoulder Elbow Surg. 2016 Nov 22. [Epub ahead of print] doi: 10.1016/j. jse.2016.09.053.

27. Terrier A, Ston J, Farron A. Importance of a three-dimensional measure of humeral head subluxation in osteoarthritic shoulders. J Shoulder Elbow Surg. 2015;24:295-301.

28. von Eisenhart-Rothe R, Muller-Gerbl M, Wiedemann E, Englmeier KH, Graichen H. Functional malcentering of the humeral head and asymmetric long-term stress on the glenoid: potential reasons for glenoid loosening in total shoulder arthroplasty. $J$ Shoulder Elbow Surg. 2008;17:695-702.

29. Walch G, Badet R, Boulahia A, Khoury A. Morphologic study of the glenoid in primary glenohumeral osteoarthritis. J Arthroplasty. 1999;14:756-760.

30. Walch G, Boulahia A, Boileau P, Kempf JF. Primary glenohumeral osteoarthritis: clinical and radiographic classification. The Aequalis Group. Acta Orthop Belg. 1998;64(suppl 2):46-52. 